\title{
Diagnosis of cobalamin $\mathrm{C}$ deficiency with renal abnormality from onset in a Chinese child by next generation sequencing: A case report
}

\author{
QIUXIA CHEN, HUAYING BAO, HONGMEI WU, SANLONG ZHAO, SONGMING HUANG and FEI ZHAO \\ Department of Nephrology, Children's Hospital of Nanjing Medical University, Nanjing, Jiangsu 210008, P.R. China
}

Received September 26, 2016; Accepted April 11, 2017

DOI: $10.3892 / \mathrm{etm} .2017 .4970$

\begin{abstract}
The aim of the present study was to present the diagnosis and treatment course of a patient with cobalamin $\mathrm{C}$ deficiency (cblC) hospitalized with renal function abnormality from the onset. A female, 7-year-old patient who presented with a cough and progressive dyspnea for 1 day was admitted to the Children's Hospital of Nanjing Medical University (Nanjing, China). A routine clinical examination was performed, including physical examination, routine blood and urine tests, blood gas analysis, computed tomography scans of the head, chest and abdomen, electrocardiogram, echocardiography and abdominal ultrasonography. In addition, laboratory tests were performed, including tests for viral infection and markers of autoimmunity, humoral immunity, myocardial enzymes and tumors. Tandem mass analysis and renal biopsy were conducted. Next generation sequencing (NGS) was performed to identify mutated genes, and structural investigation was conducted to identify the key residue mutations in the patient. Routine clinical examination revealed that the patient had multiple organ failure, indicating the presence of metabolic disease. Tandem mass analysis and renal biopsy also indicated that the patient had methylmalonic acidemia (MMA) and thrombotic microangiopathy combined with focal renal cortical necrosis. Furthermore, next-generation sequencing identified the presence of two heterozygous mutations in the MMA cblC type with homocystinuria (MMACHC) gene. Structural analysis demonstrated that the two mutations were in key components of the MMACHC protein. The patient was finally diagnosed with cblC according to the results obtained. In conclusion, NGS may aid in the diagnosis and therapeutic management of cblC with renal abnormality from the onset in children.
\end{abstract}

Correspondence to: Dr Fei Zhao, Department of Nephrology, Children's Hospital of Nanjing Medical University, 72 Guangzhou Road, Nanjing, Jiangsu 210008, P.R. China

E-mail: zhaofei75@163.com

Key words: cobalamin C, methylmalonic acidemia, homocystinuria, renal failure

\section{Introduction}

Methylmalonic acidemia (MMA), also known as methylmalonic aciduria, is a common organic acidemia. It is an autosomal recessive (AR) inherited disease caused by a deficiency or mutations in methylmalonyl-coA mutase or its coenzyme adenosylcobalamin (AdoCbl) (1). These mutations may induce abnormal accumulations of methylmalonic acid, methyl propionate, citric acid and other organic acids, thereby leading to multiple organ injuries (1). Inherited disorders of intracellular cobalamin (cbl) metabolism are classified into nine genetic complementation groups (cblA-G, cblJ and cblX) (2). Isolated MMA is characterized by MMA without hyperhomocystinuria and is caused by a deficiency of methylmalonyl-CoA mutase (mut -or mut 0) (3).

$\mathrm{CblC}$ is the most common congenital disorder of intracellular cobalamin metabolism and is characterized by combined MMA and homocystinuria (4). It is clinically divided into two main forms according to the age of onset $(5,6)$. Early-onset patients typically present with retarded growth, feeding difficulties and respiratory distress within the first two months of life, while late-onset patients usually exhibit fatigue, delirium, tonic spasms or dementia at any time in life after the age of $4(5,6)$. More than half of cblC patients have metabolic acidosis that manifests as ketonemia, ketonuria and hyperammonemia accompanied by blood abnormalities such as megaloblastic and giant cell anemia (7).

Since the discovery of the DNA double helix structure and the development of DNA sequencing methods, including Sanger sequencing, the area of genome sequencing has advanced with regard to research applications and clinical diagnostics. The advent of next generation sequencing (NGS) has revolutionized the field of genomic medicine and enabled efficient and accurate analysis of associated genes in hereditary diseases, including renal pathologies and neuromuscular disorders (2-4,6,7). Using a small amount of DNA from patients, multigene mutational profiling may be conducted with bioinformatics tools, which is now widely used in the identification of causative genes and undiagnosed early-onset disorders $(8,9)$.

$\mathrm{CblC}$ is caused by mutations in the MMACHC gene, which may result in cytoplasmic and lysosomal metabolism disorders, as well as deficiencies in the synthesis of AdoCbl and methylcobalamin ( $\mathrm{MeCbl})(10)$. The most common clinical manifestations of cblC are repeated vomiting, lethargy and 
other neurological symptoms, while cases of renal dysfunction are rare and difficult to diagnose. The current study presents a case of a patient with cblC with renal function abnormality from the onset. In addition, the molecular mechanism of MMA was investigated by analyzing the reported crystal structure of MMACHC to provide greater insight into the disease.

\section{Case report}

Patient data. A 7-year-old girl, who had presented with vomiting for two days, and cough and progressive dyspnea for one day, was admitted to the Children's Hospital of Nanjing Medical University on February 2015. Two days before admission, the patient developed the symptom of frequent vomiting, and was vomiting moderate amounts of non-spraying, bile-like liquid more than 10 times per day. No hematemesis or diarrhea were present. One day later, the patient developed a paroxysmal cough and dyspnea, with a small amount of sputum but without panting, fever or orthopnoea. The current study was approved by the Medical Ethics Committee of the Nanjing Children's Hospital Affiliated to Nanjing Medical University. Informed consent was obtained from the parents of the patient.

Routine clinical examination. Upon admission, a series of routine examinations were performed, including physical examination, routine blood examination, urinalysis, blood gas analysis, computed tomography (CT) scans of the head, chest and abdomen, electrocardiogram, echocardiography and abdominal ultrasonography. In addition, laboratory tests were performed, including tests for contagion (hepatitis B surface antigen, anti-hepatitis $\mathrm{C}$ virus, anti-human immunodeficiency virus and anti-syphilis) and markers of autoimmunity (anti-SM, anti-double stranded DNA, antinuclear antibody, anti-neutrophil cytoplasmic antibodies, myeloperoxidase, proteinase 3 and anti-cardiolipin), humoral immunity (blood $\mathrm{IgG}, \mathrm{Ig} \mathrm{A}$ and IgM, complement $\mathrm{C} 3$ and $\mathrm{C} 4)$, myocardial enzymes (lactate dehydrogenase, creatine kinase (CK), CK myoglobin bound and hydroxybutyrate dehydrogenase), and tumors ( $\alpha$-fetoprotein, carcinoembryonic antigen, neuron-specific enolase and cancer antigen 19-9).

Liquid chromatography-tandem mass spectrometry (LC-MS/MS). To analyze compounds in the serum of the patient, LC-MS/MS was performed. For sample preparation, $2 \mathrm{ml}$ peripheral blood was centrifuged at $780 \mathrm{~g}$ for $10 \mathrm{~min}$ at $4^{\circ} \mathrm{C}$ and the supernatant of serum was extracted. Subsequently, $10 \mu \mathrm{l}$ serum was added to $230 \mu \mathrm{l}$ isotope internal standard, followed by vortex at $2,000 \mathrm{r} / \mathrm{min}$ for $30 \mathrm{sec}$. The mixture was then subjected to centrifugation at $13,800 \mathrm{x} g$ at room temperature for $10 \mathrm{~min}$ and $200 \mu \mathrm{l}$ supernatant was transferred into a clean centrifuge tube for drying with nitrogen at $55^{\circ} \mathrm{C}$, followed by redissolving in $0.1 \%$ formic acid and vortex at $1,500 \mathrm{r} / \mathrm{min}$ for $1 \mathrm{~min}$. Finally, after ultrasonication at room temperature for $1 \mathrm{~min}$, the sample was subjected to centrifugation at $13,800 \mathrm{~g}$ at room temperature for $1 \mathrm{~min}$, and the supernatant was extracted. For sample detection, API 3200 ${ }^{\mathrm{TM}}$ LC/MS/MS (AB SCIEX, Framingham, MA, USA) was applied and reactive ion scanning was selected in positive ion mode by electrospray ionization to quantify compounds. The parameters used were as follows: Monitoring transitions assessed $(\mathrm{m} / \mathrm{z})$ for methionine (Met, 153.1-107.1), free carnitine (C0, 171.1-103.1), acetylcarnitine (C2, 207.1-85.0), and propionyl carnitine $(\mathrm{C} 3,221.1-80.5)$; nitrogen gas temperature was set at $350^{\circ} \mathrm{C}$; nebulizer pressure (psi) was set at $30 \mathrm{Lpm}$; and the flow rate was $0.012-0.7 \mathrm{ml} / \mathrm{min}$. The internal standard method (11) was used to quantify the analyte and the peak intensity ratio between the target analyte and the internal standard was the key parameter of the results. The compounds were calculated with the following formula:

$$
C_{\text {analyte }}=\frac{I_{\text {analyte }} \cdot V_{e x} \cdot C_{\text {is }}}{V_{B S} \cdot I_{I S} \cdot R R F} .
$$

Where $\mathrm{C}$ analyte was the target analyte concentration $(\mu \mathrm{M})$; I analyte indicated the target analyte response strength; $\mathrm{V}$ ex indicated the sample extraction volume $(\mu \mathrm{l}) ; \mathrm{C}$ is, represented the standard solution concentration $(\mu \mathrm{M})$ VBS indicated the sample usage (blood volume $3.1 \mu \mathrm{l}$ ); IIS represented the internal standard response intensity; and RRF represented the relative response factor.

Renal biopsy. Ultrasound-guided kidney puncture biopsy was performed and the tissue was subjected to pathological examination. The slices of each staining all were viewed under a light microscope (DM3000 LED; Leica, Wetzlar, Germany). Routine hematoxylin and eosin (H\&E), Periodic acid-Schiff (PAS) staining and Masson's trichrome staining were applied. For H\&E staining, the isolated tissue was washed three times with normal saline and fixed with $10 \%$ formalin solution for $48 \mathrm{~h}$ at $4^{\circ} \mathrm{C}$. Dehydration was performed with ethanol. The tissue was then embedded with paraffin, cut into $4-\mu \mathrm{m}$ sections and stained with H\&E.

For PAS staining, the tissue preparation steps were the same as that of the H\&E staining. Paraffin-embedded slices were deparaffinized, hydrated with distilled water and oxidized at $40^{\circ} \mathrm{C}$ in $0.5 \%$ periodic acid solution for $10 \mathrm{~min}$. The sections were washed with distilled water and placed in Schiff reagent. Sections were then washed in distilled water, counterstained with Mayer's hematoxylin, washed in distilled water, dehydrated with gradient ethanol and sealed using a synthetic mounting medium.

For Masson's trichrome staining, deparaffinized and rehydrated sections were fixed with Bouin's solution (a mixture containing $1.22 \%$ picric acid, $75 \mathrm{ml}$; formalin, $25 \mathrm{ml}$; and acetic acid, $5 \mathrm{ml}$ ) at $56^{\circ} \mathrm{C}$ for $1 \mathrm{~h}$. The sections were then stained with modified Weigert's iron hematoxylin, $0.5 \%$ hydrochloric acid in $70 \%$ ethanol, Biebrich scarlet-acid fuchsin solution, phosphomolybdic-phosphotungstic acid solution, aniline blue solution and acetic acid solution successively. After dehydration in graded ethanol and clearing in graded xylene, the sections were mounted with synthetic resin. Focal coagulation necrosis of glomerulus was defined as the percentage of the focal coagulation necrosis glomerular to total glomerular at high power magnification (x400).

Genetic sequencing. DNA sample sequencing were submitted to the Clinical Laboratory Center of the Beijing Genomics Institute (Beijing, China) for gene detection. Briefly, genomic DNA from $2 \mathrm{ml}$ peripheral blood was extracted using a BloodGen Midi kit (CWBio Co., Ltd., Beijing, China), according to the manufacturer's protocol. DNA was sheared to $200-400$ bp by $\mathrm{S} 220$ 
sonication (Covaris, Woburn, MA, USA) and hybridized with a NimbleGen 2.0 Sequence Capture array (Roche Diagnostics, Basel, Switzerland). The captured DNA was subjected to exonic DNA enrichment (MyGenostics, Inc., Beijing, China) following the manufacturer's protocol. In brief, $1 \mu \mathrm{g}$ DNA library was mixed with Buffer BL and GenCap gene panel probe (MyGenostics, Inc.), heated at $95^{\circ} \mathrm{C}$ for $7 \mathrm{~min}$ and $65^{\circ} \mathrm{C}$ for $2 \mathrm{~min}$ on a polymerase chain reaction (PCR) machine. Subsequently, $23 \mu \mathrm{l}$ of the $65^{\circ} \mathrm{C}$ pre-warmed Buffer HY (MyGenostics, Inc.) was added to the mix, and the mixture was held at $65^{\circ} \mathrm{C}$ for $22 \mathrm{~h}$ for hybridization. MyOne beads (50 $\mu \mathrm{l}$; Life Technology; Thermo Fisher Scientific, Inc., Waltham, MA, USA) was washed in $500 \mu 11 \mathrm{X}$ binding buffer 3 times and resuspended in $80 \mu 11 \mathrm{X}$ binding buffer. A total of $64 \mu \mathrm{l} 2 \mathrm{X}$ binding buffer was added to the hybrid mix and transferred to the tube with $80 \mu \mathrm{l}$ MyOne beads. The mix was rotated for $1 \mathrm{~h}$ at room temperature on a rotator. The beads were then washed with WB1 buffer at room temperature for 15 min once and $\mathrm{WB} 3$ buffer at $65^{\circ} \mathrm{C}$ for 15 min 3 times. The bound DNA was then eluted using elution buffer. The eluted DNA was amplified for 15 cycles using the following program: $98^{\circ} \mathrm{C}$ for $30 \mathrm{sec}(1 \mathrm{cycle}) ; 98^{\circ} \mathrm{C}$ for $25 \mathrm{sec}$, $65^{\circ} \mathrm{CC}$ for $30 \mathrm{sec}, 72^{\circ} \mathrm{C}$ for $30 \mathrm{sec}\left(15\right.$ cycles); and $72^{\circ} \mathrm{C}$ for $5 \mathrm{~min}$ (1 cycle). The PCR product was purified using SPRI beads (Beckman Coulter, Inc., Brea, CA, USA) according to manufacturer's protocol. The libraries were tested for size distribution and concentration using an Agilent Bioanalyzer 2100 (Agilent Technologies, Inc., Santa Clara, CA, USA) according to the manufacturer's protocol. The samples were then sequenced on an Illumina Hiseq2500 (Illumina, Inc., San Diego, CA, USA). Raw image files were processed using BclToFastq software (version number, 2.18.0.12; Illumina, Inc.) for base calling and raw data generation. Low-quality variations were filtered out using a quality score $\geq 20$. The sequencing reads were aligned to the NCBI human reference genome (HG-19) using Burrows-Wheeler Aligner software (http://maq.sourceforge.net; version number 0.7.15) as described previously (12). Genome Analysis Toolkit software (GATK-3.7 software; Broad Institute, Cambridge, MA, USA) was used to analyze single nucleotide polymorphisms and insertion-deletions in the sequences. Sanger sequencing was used to confirm the mutations identified in the cohort. The PCR products were sequenced using an ABI 3730XL (Thermo Fisher Scientific, Inc., Waltham, MA, USA) and analyzed using DNASTAR 5.0 software (DNASTAR, Inc., Madison, WI, USA).

Structural investigation. The reported mutational sites of MMACHC were identified using the online sever of the Human Gene Mutation Database (www.hgmd.cf.ac.uk). The effect of the missense mutation was predicted using PolyPhen-2 (http:/genetics.bwh.harvard.edu/pph2/). The crystal structure of MMACHC (1-238) was downloaded from the Protein Data Bank (PDB) database (www.rcsb.org/pdb) in PDB format (code 3SC0). Structural figures were prepared and displayed using PyMol v1.8.0.7 (www.pymol.org). The MeCbl ligand and key residues were indicated as sticks.

General condition of the patient. The patient was the first and only girl of non-consanguineous parents, with no abnormalities in childbirth or feeding history. At 4 years old, the patient presented with slightly delayed growth and development.
On the first day of her admission, the patient's weight and height were $17 \mathrm{~kg}$ (normal, $21 \mathrm{~kg}-22 \mathrm{~kg}$ ) and $120 \mathrm{~cm}$ (normal, $122.1-135.5 \mathrm{~cm})$, respectively. The patient's parents denied any family history of hereditary disease. Physical examination indicated that the patient was conscious but slightly unresponsive. The patient exhibited regular breathing, with no rash or cyanosis. Phlegm in the throat and scattered bilateral pulmonary phlegm was audible. There was a grade $2 / 6$ murmur in the precordium. The liver was palpable $4 \mathrm{~cm}$ below the right costal margin. No abnormal physical signs were identified in the limbs or nervous system.

Routine clinical examination results. To understand the patient's condition, a series of routine clinical examinations were performed. Blood routine examination revealed that the white blood cell count was $17.4 \times 10^{9} / 1$ (normal range, 4-10x $10^{9} / 1$ ) and hemoglobin was $108 \mathrm{~g} / 1$ (normal range, 110-160 g/l). Blood biochemistry examination illustrated that urea was $19.59 \mathrm{mmol} / 1$ (normal range, 2.9-8.2 $\mathrm{mmol} / \mathrm{l}$ ), creatinine was $185 \mu \mathrm{mol} / 1$ (normal range, 30-104 $\mu \mathrm{mol} / \mathrm{l}$ ), potassium was $6.36 \mathrm{mmol} / \mathrm{l}$ (normal range, 3.5-5.5 mmol/l) and sodium was $115.7 \mathrm{mmol} / \mathrm{l}$ (normal range, 136-145 $\mathrm{mmol} / \mathrm{l}$ ), and no other abnormalities were identified. The endogenous creatinine clearance rate was $31.5 \mathrm{ml} / \mathrm{min} / 1.73 \mathrm{~m}^{2}$ (normal range, $>90 \mathrm{ml} / \mathrm{min} / 1.73 \mathrm{~m}^{2}$ ), indicating that renal function was impaired. Urinalysis showed that the urine protein value was $3+$ (normal range, negative) with red blood cells at a value at +++ HP (30 red blood cells per high power field; normal range, negative) under microscopic examination. Blood gas analysis showed that the $\mathrm{pH}$ value was 7.432 (normal range, 7.35-7.48), partial pressure of oxygen was $68.4 \mathrm{mmHg}$ (normal range, 83-108 $\mathrm{mmHg}$ ), arterial base excess was $-8.1 \mathrm{mmol} / 1$ (normal range, -2.7-2.5 $\mathrm{mmol} / \mathrm{l}$ ), lactic acid was $1.4 \mathrm{mmol} / 1$ (normal range, 0 ) and bicarbonate was $18.5 \mathrm{mmol} / 1$ (normal range, 21-28 mmol/l). These results indicated metabolic acidosis. Chest CT identified diffuse lesions in both lungs. The skull CT scan was normal, indicating that no intracranial space-occupying had occurred. Abdominal CT identified pelvic effusion, and the abdominal muscle exhibited diffuse calcification, as depicted in Fig. 1. Abdominal ultrasonography identified mild swelling in the liver, with thickening of the gallbladder wall, hydrothorax in both sides and ascites. The electrocardiogram result indicated that the patient had sinus tachycardia with an AVr R/Q ratio $>1$, and the heart axis was at a right slant. Cardiac ultrasonography showed that left ventricular systolic ejection fraction was $49 \%$ (normal, $>55 \%$ ) and $\mathrm{E} / \mathrm{A}<1$ (normal, $>1$ ), suggesting cardiac insufficiency and left ventricular systolic dysfunction. Blood pressure fell to $55 / 36 \mathrm{mmHg}$ (normal value, $\sim 95 / 60 \mathrm{mmHg}$ ) and at day 3 after admission, the patient became comatose with anuria and azotemia (urea and creatinine were $29.9 \mathrm{mmol} / 1$ and $384 \mu \mathrm{mol} / 1$, respectively) accompanied by severe disorders of electrolyte and acid-base balance ( $\mathrm{pH} 7.051$ and lactic acid $4.1 \mathrm{mmol} / \mathrm{l}$ ). Liver dysfunction was also observed (concentrations of alanine aminotransferase, aspartate aminotransferase and lactic dehydrogenase were 1,870 (normal range, 5-40 U/1), 1,250 (normal range, 5-40 U/1) and 2,370 (normal range, 80-285 U/1) U/1, respectively, along with many other symptoms such as coagulation disorders (prothrombin time. $16.5 \mathrm{sec}$; international normalized ratio, 1.40; partial prothrombin time, $89.1 \mathrm{sec}$; fibrin, $1.53 \mathrm{~g} / \mathrm{l})$, gastrointestinal hemorrhage, 


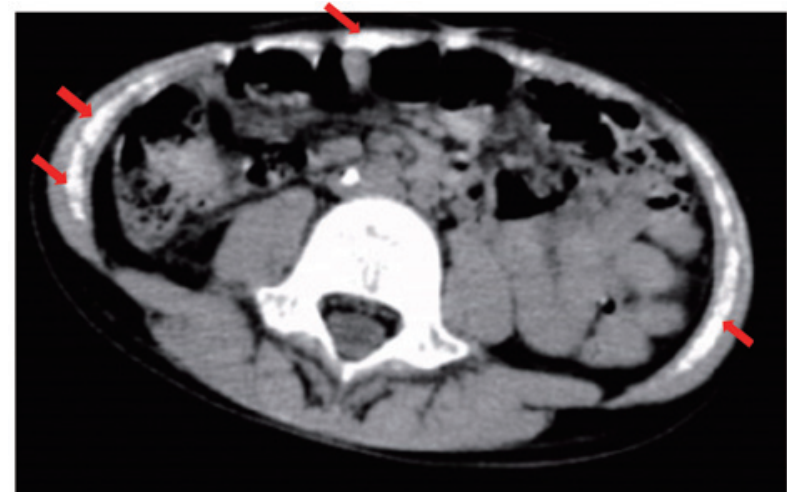

Figure 1. Abdominal computed tomography scan of the patient upon admission. The scan identified diffuse calcification of abdominal muscles (arrows).

hypertension (blood pressure, 171/104 $\mathrm{mmHg}$ ), hyperglycemia (glucose levels after fasting, $22.2 \mathrm{mmol} / \mathrm{l}$; normal fasting range, 3.9-6.1 mmol/l), anemia (hemoglobin concentration of $68 \mathrm{~g} / \mathrm{l}$ (normal range, 110-160 g/l), and low platelet count (84 x10 $/ 1$; normal range, $\left.100-300 \times 10^{9} / 1\right)$.

Mechanical ventilation was applied on the day she was hospitalized in February 2015. Anti-infection was achieved by intravenous drip of meropenem $(20 \mathrm{mg} / \mathrm{kg}$ every $12 \mathrm{~h}$; Sumitomo Dainippon Pharma, Co., Ltd., Osaka, Japan) and vancomycin $(10 \mathrm{mg} / \mathrm{kg}$ once daily; VIANEX S.A., Athens, Greece). Intravenous micro pumps of dopamine $(5 \mu \mathrm{g} / \mathrm{kg} / \mathrm{min}$; Yabang Pharmaceutical Co., Ltd., Changzhou, China) and dobutamine (3 $\mu \mathrm{g} / \mathrm{kg} / \mathrm{min}$; Zhejiang Ruixin Pharmaceutical Co., Ltd., Lishui, China) were used to improve the microcirculation and maintain blood pressure. Intravenous drip of omeprazole $(0.8 \mathrm{mg} / \mathrm{kg}$ once daily; Hangzhou Ausia Biological Technology Co., Ltd., Zhejiang, China) was applied to inhibit gastric acid secretion and sodium bicarbonate was taken for correcting acidosis and electrolyte supplement. Day 2 after admission, in addition to the previously mentioned drug treatments, the following treatment was undertaken: Intravenous drip of fructose diphosphate sodium $(2 \mathrm{~g} /$ day; Hainan GM SANYO Pharmaceutical Co., Ltd., Hainan, China) was applied for myocardium nutrition and mouse nerve growth factor (30 $\mu \mathrm{g}$ once daily; Wuhan Haite Biological Pharmacy Co., Ltd., Hubei, China) intramuscular injection was performed for brain nutrition. Intravenous drip of voriconazole ( $3 \mathrm{mg} / \mathrm{kg}$ every $12 \mathrm{~h}$; Zhuhai Ebang Pharmaceutical Co., Ltd., Zhuhai, China) was used for antifungal therapy on Feb, 27. From the third day after admission, additional therapy was administered. Antiviral (acyclovir; intravenous drip; 5 mg/kg every 12 h; Hu Bei Keyi Pharmaceutical Co., Ltd., Hubei, China) and anti-inflammatory (dexamethasone; 5 mg/day; intravenous drip; Hu Bei Keyi Pharmaceutic Co., Ltd.) treatments were performed. In addition, 3 days after admission, hyperglycaemia (glucose levels, $22.2 \mathrm{mmol} / 1$, normal fasting range, 3.9-6.1 mmol/l) was observed and insulin (Novo Nordisk A/S; Bagsvaerd, Denmark; $1.7 \mathrm{U} / \mathrm{h}$ for $3 \mathrm{~h}$ using a micro pump) was used to lower blood sugar. Hemodialysis was performed every other day and $\gamma$-globulin (Human Immunoglobulin ( $\mathrm{pH}$ 4) was applied by intravenous injection (one treatment of $10 \mathrm{~g}$ every day for 2 days, Sichuan Yuanda Shuyang Pharmaceutical Co., Ltd., Chengdu, China) was applied. At 15 days after admission, the patient regained consciousness, accompanied by heart function improvement (cardiac function and myocardial enzyme were normal 12 days after admission) and breathing without a ventilator. However, the patient presented with continued oliguria (urine output, 100-300 ml/day), renal dysfunction (20 days after admission; urea nitrogen, $15.71 \mathrm{mmol} / \mathrm{l}$; creatinine, $287 \mathrm{umol} / \mathrm{l}$ ) and high blood pressure (blood pressure, 173/121 $\mathrm{mmHg}$ ); therefore, the patient was transferred to nephrology to undertake renal biopsy. The aforementioned results indicated that the patient had multiple organ failure, which indicated that metabolic disease should be taken into consideration.

Quantitative analysis results. Blood compounds were measured to provide biochemical indicators of amino acid-related metabolism diseases and assist clinical diagnosis of organ pathological changes by blood tandem mass spectrometry. The results indicated that blood methionine was $5.35 \mu \mathrm{mol} / 1$ (normal range, 9.0-40.0 $\mu \mathrm{mol} / \mathrm{l}$ ), propionyl-carnitine (C3) was $5.38 \mu \mathrm{mol} / 1$ (normal range, $0.5-4.0 \mu \mathrm{mol} / \mathrm{l}$ ) and the ratio of $\mathrm{C} 3$ to acetylcarnitine (C2) was 0.33 (normal range, 0.03-0.25). At 35 days after admission, after intramuscular injection of cyanocobalamin vitamin B12 (Shanghai Xinyi Jinzhu Pharmaceutical Co., Ltd., Shanghai, China) at $1 \mathrm{mg} /$ day for 5 days, the indicators were reexamined and the results were as follows: Methionine was $4.94 \mu \mathrm{mol} / 1$, C3 was $2.73 \mu \mathrm{mol} / 1$ and homocysteine concentration was $100 \mu \mathrm{mol} / 1$ (normal range, $0-13 \mu \mathrm{mol} / \mathrm{l}$ ). Analysis of organic acids was also performed. The main index of methylmalonic acid was 8.75 (normal range, 0.3-3.6) and the auxiliary index of 2-methyl-3-hydroxybutyric acid was 8.95 (normal range, 0.1-0.3). On the basis of these results, the patient was diagnosed with combined MMA and homocystinuria.

Renal biopsy examination results. To investigate pathological changes of the kidney, renal biopsy examination was performed, including H\&E, PAS and Masson staining (Fig. 2). It was observed that $46 \%$ of the glomerulus exhibited focal coagulation necrosis (Fig. 2A) and thrombogenesis was observed in partial glomerular capillaries as well as afferent glomerular arterioles (Fig. 2B). The number of cells in the glomerulus increased, with diffuse proliferation and segmental swelling of glomerular endothelial cells. The subcutaneous tissue was loose, and the basement membrane had thickened and twisted (Fig. 2C). PAS stain showed segmental sclerosis of the glomerulus, glomerular adhesions and glomerulosclerosis (Fig. 2D-F). As illustrated in Fig. 2G, flat renal tubular epithelial cells with the absence of the brush border were revealed by H\&E. PAS staining demonstrated granular degeneration and vacuolar degeneration of the renal tubular epithelial cells (Fig. 2H-J). These results indicated that the patient had thrombotic microangiopathy combined with focal renal cortical necrosis.

Genetic sequencing results. To identify genetic mutations in the patient, high-throughput sequencing uncovered the presence of two heterozygous mutations in the MMACHC gene (Fig. 3). One was a missense caused by mutation of the 80th base from adenine to guanine (c.80A $>\mathrm{G}$ ) as well as its coding amino acid from glutamine to arginine (p.Gln27Arg). The other was a nonsense mutation, which occurred in the 609th base through replacement of guanine to adenine (c.609G $>$ A) and resulted in the termination of protein translation (p.Trp203Ter). These 
A

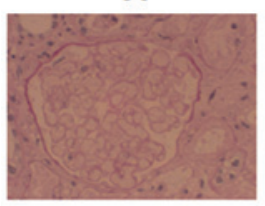

$\mathbf{F}$

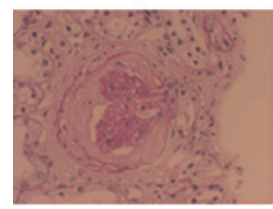

B

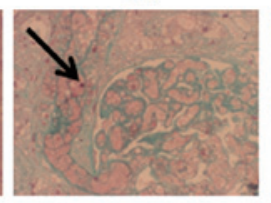

G

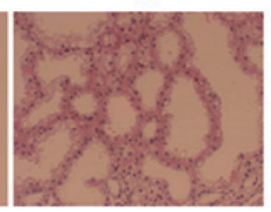

C

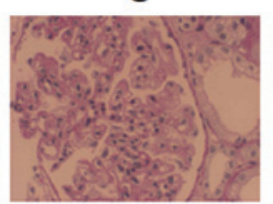

H

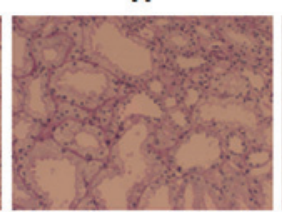

D

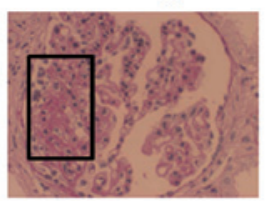

I

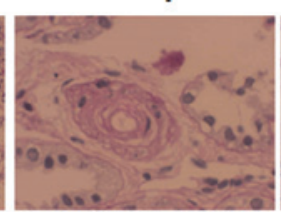

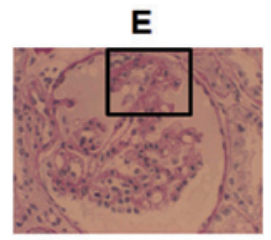

J

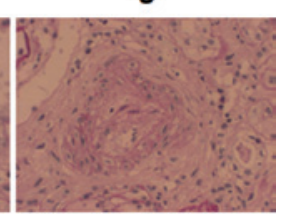

Figure 2. Renal biopsy examination using H\&E, PAS and Masson's trichrome staining (magnification, $\mathrm{x} 400$ ). (A) PAS stain indicating coagulation necrosis of the glomerulus. (B) Masson's trichrome stain indicating thrombosis in the afferent arterioles and capillary lumen (black arrow; the surrounding region; green, immune complex; and pink, thrombus). (C) PAS stain indicating abnormalities in glomerular volume and endothelial cells. (D) PAS stain indicating segmental sclerosis of the glomerulus (black square). (E) PAS stain indicating balloon adhesions (black square). (F) PAS stain indicating glomerulosclerosis. (G) H\&E stain indicating flat renal tubular epithelial cells with absence of the brush border. $(\mathrm{H})$ PAS stain indicating granular degeneration and vacuolar degeneration of the renal tubular epithelial cells. (I) and (J) PAS stain indicating endothelial hyperplasia, swelling, intimal edema and vascular stenosis in the interstitial arterioles. H\&E, hematoxylin and eosin; PAS, Periodic acid-Schiff.

mutations were verified by Sanger sequencing (Fig. 3). These data demonstrated that the disease was caused by compound heterozygous mutations in the MMACHC gene.

Effect of patient mutations on MMAHCH protein structure. Structural analysis of the mutation sites was performed based on the reported crystal structure of MMAHCH in complex with $\mathrm{MeCbl}$ (10). The effect of the c.80A $>\mathrm{G}$ missense mutation was predicted with the online sever PolyPhen- 2 prior to structural investigation. This mutation was predicted to be benign with a score of 0.022 (sensitivity, 0.95 ; specificity, 0.80). The protein structure, which is composed of 238 amino acids (the C-terminal $\sim 40$ residues are highly unstructured and absent) has a flavin reductase fold that binds to $\mathrm{MeCbl}$ in a base-off form (Fig. 4). According to previous results (13), most of the pathological mutations in MMAHCH occur in positions important for cobalamin binding or structural integrity. Fig. 4A presents mutation sites of the $\mathrm{MMAHCH}$ protein identified in the patient. Gln27 is in the core region of the N-terminal module and interacts with Trp30, Pro172 and Leu174 through hydrogen bond interactions, which is critical for structural stability of the protein (Fig. 4B). The other mutant site p.Trp203Ter was predicted to be close to the C-terminal, where it may lead to the production of a premature protein that lacks key sites for binding to $\mathrm{MeCbl}$, as well as causing instability and dysfunction of the MMACHC protein (Fig. 4C). Overall, the PolyPhen-2 result indicated that a single mutation of Gln27Arg may not induce symptoms; however, the two mutations detected by structural investigation of the MMAHCH protein may have exerted critical impacts on the function of MMAHCH. Thus, the patient's disorder was concluded to be caused by genetic mutations. The patient was transferred to another hospital 42 days after admission to our hospital and a 6-month follow-up showed that there was no need for dialysis; however, she was in the renal failure stage.

\section{Discussion}

The patient in the present study was acute onset with clinical manifestations of hemoglobin decrease, thrombocytopenia and multiple organ failure. As renal biopsy revealed thrombotic microangiopathy (TMA) and focal cortical necrosis, it was initially suspected that the patient had TMA, since all the pathological features were typical of TMA. However, the color of urine and serum bilirubin were both normal during the course of treatment, and no broken red blood cells were detected in the peripheral blood, thus indicating that a diagnosis of hemolytic uremic syndrome (HUS) or thrombotic thrombocytopenic purpura was incorrect. Following combined urine and renal function analyses, renal biopsy and chest and abdomen $\mathrm{CT}$, primary renal diseases and polymyositis were excluded. It was suspected that the symptoms were caused by inherited metabolic disease, as previous research has indicated that metabolic disorders of cobalamin are the third most common differential diagnosis of atypical HUS (14). Based on these findings, further screening of blood and urine metabolism and gene sequencing were performed. Blood mass spectrometry indicated that $\mathrm{C} 3$, $\mathrm{C} 3 / \mathrm{C} 2$ and homocysteine increased markedly, while methionine decreased slightly. Combined with markedly increased methylmalonic acid in the urine, the patient was diagnosed with MMA, type cblC. The decline in $\mathrm{C} 3 / \mathrm{C} 2$ following injection of cobalamin for 5 days verified the diagnosis.

MMA combined with homocystinuria is the most common type of MMA and accounts for $80 \%$ of MMA cases in China (5). Subtype cblC is the predominant diagnosis among all MMA patients in China (5). Most patients with cblC develop systemic symptoms at an early age (early-onset, $\leq 1$ year old), while late-onset patients ( $>1$ year old) typically develop symptoms after 4 years of age and exhibit relatively mild physical symptoms. A previous statistical report revealed that late-onset cblC accounts for only $10 \%$ of all cblC patients (13). The 7-year-old patient in the present study was diagnosed as late-onset cblC and presented clinical features of repeated vomiting, drowsiness, coma, multiple organ failure and metabolic acidosis, all of which were consistent with manifestations of MMA (15). The onset age was relatively old; however, the patient developed renal damage immediately after hospitalization, which is rare, and thus the diagnostic process was difficult.

Previously reported manifestations of MMA associated with renal damage are primarily proteinuria, hematuria, 
A

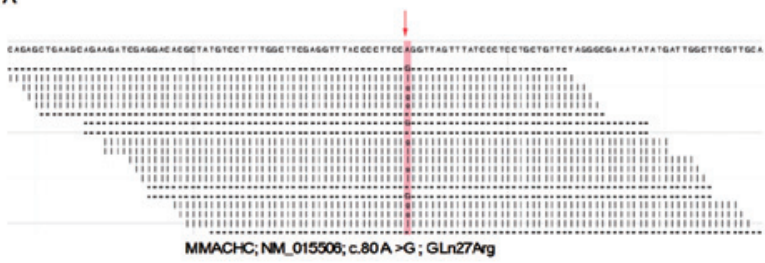

MMACHC; NeM_015506; C.80A >G; GLIR27ANG

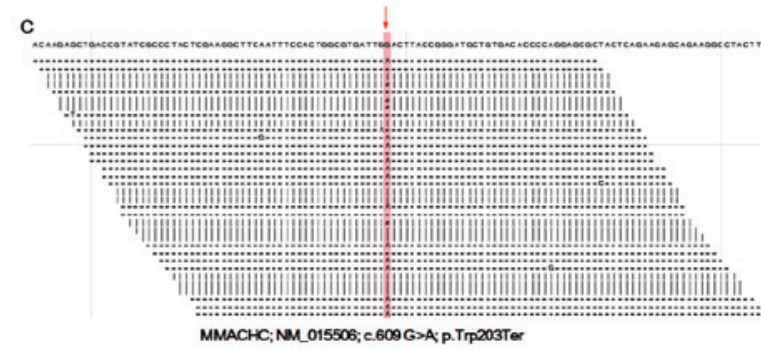

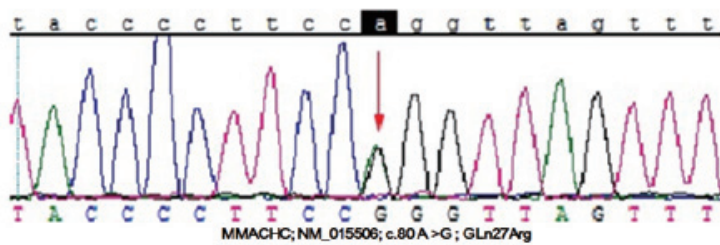

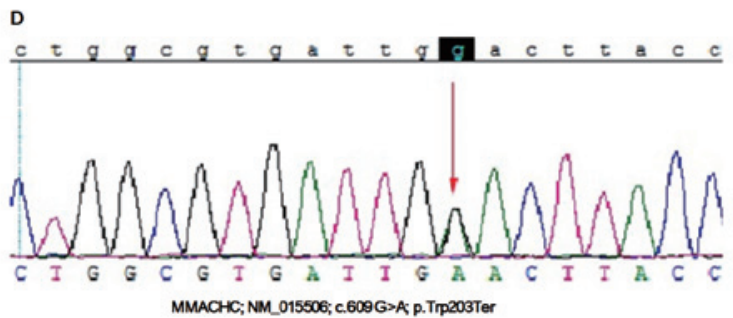

Figure 3. (A-D) Sequencing analysis. (A and C) Next generation sequencing and (B and D) Sanger sequencing results revealed A/G heterozygous peaks, indicating (A and B) c.80A $>$ G (Gln27Arg) and (C and D) c.609G $>$ A (Trp203Ter) heterozygous mutations. The grey lines indicated that the nucleic acids were in line with the reference sequence.
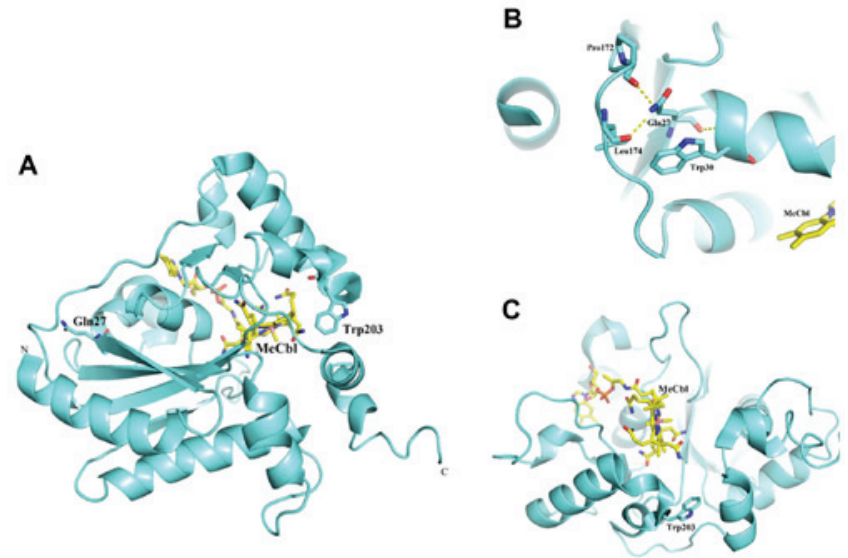

Figure 4. Crystal structure of human MMACHC with MeCbl. MMACHC is displayed in cyan and MeCbl in yellow. Mutation sites (Gln27 and Trp203) of the patient are shown as sticks. (A) Overall structure of the complex. (B) Interactions of Gln27 with other amino acids. Hydrogen bonds are shown as dashed lines. (C) Structural details of Trp203. Termination of the protein due to the Trp203Ter mutation results in the loss of multiple interactions among the $\mathrm{C}$-terminal amino acids and structural instability of the protein. $\mathrm{Cbl}$, cobalamin; MeCbl, methylCbl; MMACHC, methylmalonic acidemia cblC type with homocystinuria.

edema, hypertension and kidney failure, with some patients also diagnosed with HUS (16). The majority of patients with proteinuria or hematuria alone are late-onset and have a good prognosis, while MMA associated with HUS is mostly observed as the early-onset form, which is difficult to treat and typically has a poor prognosis (17). Accumulation of toxic substances, including methylmalonic acid and propionic acid, is among the major factors that lead to kidney tissue damage, as these toxicants may induce mitochondrial dysfunction and oxidative stress (18-21). The diagnosis and treatment course of patients with MMA could be improved. For instance, it may be beneficial to perform full examinations for inherited metabolic diseases in patients with growth retardation, acute or chronic encephalopathy, metabolic acidosis and multi-system damage. Furthermore, it has been demonstrated that an increase in both urinary methylmalonic acid and $\mathrm{C} 3 / \mathrm{C} 2$ in the serum are highly specific for MMA, and thus a combination of these diagnostic methods may reduce misdiagnosis and rate of missed diagnosis (15). For patients with increased urinary methylmalonic acid or serum $\mathrm{C} 3 / \mathrm{C} 2$, repeated inspections should be performed and genetic testing may be required to make a definitive diagnosis.

The patient in the current study was cblC type with two heterozygous mutation sites in the MMACHC gene. The MMACHC gene maps to chromosome region 1 p34.1 and consists of 5 exons, which encode a protein of 282 amino acids in length with a predicted molecular weight of $31.7 \mathrm{kDa}(21)$. More than 70 mutations have previously been identified in this gene $(22,23)$. The nonsense mutation c.609G $>$ A (p.Trp203Ter) identified in the present study may cause premature termination of protein translation and is the most frequent mutation type in China, with a frequency of 48.1-66.7\% $(22,23)$. Homozygous mutation of c.609G $>\mathrm{A}$ is typically associated with early-onset cblC deficiency with a severe phenotype and poor prognosis $(23,23)$. A mild clinical phenotype has also been observed with this mutation, though the cases were rare (24). The second heterozygous mutation site c.80A $>$ G (p.Gln27Arg) identified in the current patient produces a missense mutation, and has a relatively rare frequency of $5.1 \%$ in Chinese cblC patients (23). $\mathrm{Yu}$ et al (24) reported in a retrospective study that in 16 cases of cblC deficiency, $10(62.5 \%)$ had the c.609G $>$ A mutation with a ratio of heterozygous to homozygous of $4: 1$. Notably, one case exhibited combined heterozygous mutations of c.80A $>\mathrm{G}$ and c.609G $>$ A, which was associated with severe phenotypes and a poor therapeutic outcome, ultimately leading to patient mortality. Structural investigation of the human MMACHC protein indicated that Gln27 was located in the core region of $\mathrm{MMAHCH}$, and mutation of this residue to arginine may cause destruction of protein structural stability and thus lead to disease (10). Trp203 is located close to the C-terminal and is critical in the overall organization of the MMACHC protein (10), and thus a Trp203 mutation may result in a premature protein that lacks the C-terminal components and 
key binding sites for MeCbl. Structural investigation indicated that the two mutational sites identified in the present study were critical to the function of the MMAHCH protein, and thus were likely responsible for disease onset in the patient. To diagnose patients with compound heterozygous mutations, trio sequencing is required, though in the current study, the patient's parents did not give consent for this test to be performed. However, as MMA is an AR disease that may be identified by clinical manifestations and sequencing results, it was possible to make a definite diagnosis without tri sequencing.

Typical medications for MMA include vitamin B12, L-carnitine, betaine, calcium foliate, vitamin B8 and special milk (excluding valine, threonine and methionine) (25). It has previously been suggested that angiotensin converting enzyme inhibitor should be prescribed for MMA patients with renal damage, as this drug may aid to reduce proteinuria and promote the recovery of renal function (26). In the current study, the $\mathrm{C} 3 / \mathrm{C} 2$ value decreased after injection of vitamin $\mathrm{B} 12$, indicating that the patient was vitamin $\mathrm{B} 12$ reactive. Therefore, vitamin B12, L-carnitine, betaine and folic acid were administered. Although some functions were restored, multiple organ failure occurred during the treatment course and the patient entered a phase of long-term renal replacement therapy.

In conclusion, the current study describes the diagnosis and treatment course of a 7-year-old patient with cblC. Gene sequencing demonstrated that the patient had compound heterozygous mutations (c.609G $>\mathrm{A}$ and c.80A $>\mathrm{G}$ ) in the MMAHCH gene. Sequencing and clinical analysis was performed to investigate the pathogenesis of MMA. Based on these findings, it is proposed that clinicians, particularly nephrologists, should perform early genetic testing and treatment for patients presenting with renal damage combined with acute and chronic encephalopathy, metabolic acidosis and multiple organ lesions, so as to reduce the rate of misdiagnosis and mortality.

\section{Acknowledgements}

The present study was supported by the National Natural Science Foundation of China (grant no. 81300571).

\section{References}

1. Cantani A: Methylmalonic acidemia: Classification, diagnosis and therapy. Klin Padiatr 195: 388-393, 1983.

2. Moras E, Hosack A, Watkins D and Rosenblatt DS: Mitochondrial vitamin B12-binding proteins in patients with inborn errors of cobalamin metabolism. Mol Genet Metab 90: 140-147, 2007.

3. Ktena YP, Paul SM, Hauser NS, Sloan JL, Gropman A, Manoli I and Venditti CP: Delineating the spectrum of impairments, disabilities, and rehabilitation needs in methylmalonic acidemia (MMA). Am J Med Genet A 167A: 2075-2084, 2015.

4. Backe PH, Ytre-Arne M, Røhr AK, Brodtkorb E, Fowler B, Rootwelt $\mathrm{H}$, Bjørås $\mathrm{M}$ and Mørkrid L: Novel deletion mutation identified in a patient with late-onset combined methylmalonic acidemia and homocystinuria, cblC type. JIMD Rep 11: 79-85, 2013.

5. Zong Y, Liu N, Zhao Z and Kong X: Prenatal diagnosis using genetic sequencing and identification of a novel mutation in MMACHC. BMC Med Genet 16: 48, 2015.

6. Liu YP, Ma YY, Wu TF, Wang Q, Li XY, Ding Y, Song JQ, Huang Y and Yang YL: Abnormal findings during newborn period of 160 patients with early-onset methylmalonic aciduria, Zhonghua Er Ke Za Zhi 50: 410-414, 2012 (In Chinese).
7. Todd EJ, Yau KS, Ong R, Slee J, McGillivray G, Barnett CP, Haliloglu G, Talim B, Akcoren Z, Kariminejad A, et al: Next generation sequencing in a large cohort of patients presenting with neuromuscular disease before or at birth. Orphanet J Rare Dis 10: 148, 2015.

8. Goldmann JM, Wong WS, Pinelli M, Farrah T, Bodian D, Stittrich AB, Glusman G, Vissers LE, Hoischen A, Roach JC, et al: Parent-of-origin-specific signatures of de novo mutations. Nat Genet 48: 935-939, 2016.

9. Kitamura Y, Kondo E, Urano M, Aoki R and Saito K: Target resequencing of neuromuscular disease-related genes using next-generation sequencing for patients with undiagnosed early-onset neuromuscular disorders. J Hum Genet 61: 931-942, 2016.

10. Koutmos M, Gherasim C, Smith JL and Banerjee R: Structural basis of multifunctionality in a vitamin B12-processing enzyme. J Biol Chem 286: 29780-29787, 2011.

11. Clinical and Laboratory Standards Institute (CLSI): Newborn Screening by Tandem Mass Spectrometry. CLSI Guideline NBS04. 2nd edition. CLSI, Wayne, PA, 2017.

12. Li H and Durbin R: Fast and accurate short read alignment with Burrows-Wheeler transform. Bioinformatics 25: 1754-1760, 2009.

13. Kılıç M, Özgül RK, Dursun A, Tokatlı A, Kalkanoğlu-Sivri HS, Anlar B, Fowler B and Coşkun T: Cobalamin C defect: A patient of late-onset type with homozygous p.R132* mutation. Turk J Pediatr 55: 633-636, 2013.

14. Loirat $\mathrm{C}$ and Frémeaux-Bacchi V: Atypical hemolytic uremic syndrome. Orphanet J Rare Dis 6: 60, 2011.

15. Wu SN, Han LS, Ye J, Qiu WJ, Zhang HW, Gao XL, Wang Y, Li XY, Xu H and Gu XF: Analysis of propionylcarnitine in blood and methylmalonic acid in urine of 162 patients with methylmalonic acidemia. Zhonghua Yi Xue Za Zhi 93: 561-565, 2013 (In Chinese).

16. Liu XM, Chen Z, Yuan L, Zhou N, Jiang Y and Shen Y: Renal injury as the primary symptom in methylmalonic aciduria in 9 paediatric cases. Chin J Evidence Based Pediatrics 5: 447-451, 2010 (In Chinese).

17. Sharma AP, Greenberg CR, Prasad AN and Prasad C: Hemolytic uremic syndrome (HUS) secondary to cobalamin C (cblC) disorder. Pediatr Nephrol 22: 2097-2103, 2007.

18. Liu XR, Zhou W, Zhou N, Chen Z, Fan JF and Shen Y: A case of methylmalonic acidemia with hemolytic uremic syndrome as the main clinical manifestations. Zhonghua Lin Chuang Yi Shi Za Zhi (Dian Zi Ban) 7: 8086-8087, 2013 (In Chinese).

19. Manoli I, Sysol JR, Li L, Houillier P, Garone C, Wang C, Zerfas PM, Cusmano-Ozog K, Young S, Trivedi NS, et al: Targeting proximal tubule mitochondrial dysfunction attenuates the renal disease of methylmalonic acidemia. Proc Natl Acad Sci USA 110: 13552-13557, 2013.

20. Schuck PF, Alves L, Pettenuzzo LF, Felisberto F, Rodrigues LB, Freitas BW, Petronilho F, Dal-Pizzol F, Streck EL and Ferreira GC: Acute renal failure potentiates methylmalonate-induced oxidative stress in brain and kidney of rats. Free Radic Res 47: 233-240, 2013.

21. Gregory SG, Barlow KF, McLay KE, Kaul R, Swarbreck D, Dunham A, Scott CE, Howe KL, Woodfine K, Spencer CC, et al: The DNA sequence and biological annotation of human chromosome 1. Nature 441: 315-321, 2006.

22. Han B, Cao Z, Tian L, Zou H, Yang L, Zhu W and Liu Y: Clinical presentation, gene analysis and outcomes in young patients with early-treated combined methylmalonic acidemia and homocystinuria (cblC type) in Shandong province, China. Brain Dev 38: 491-497, 2016.

23. Liu MY, Yang YL, Chang YC, Chiang SH, Lin SP, Han LS, Qi Y, Hsiao KJ and Liu TT: Mutation spectrum of MMACHC in Chinese patients with combined methylmalonic aciduria and homocystinuria. J Hum Genet 55: 621-626, 2010.

24. $\mathrm{Yu} \mathrm{YF,} \mathrm{Li} \mathrm{F} \mathrm{and} \mathrm{Ma} \mathrm{HW:} \mathrm{Relationship} \mathrm{of} \mathrm{genotypes}$ with clinical phenotypes and outcomes in children with cobalamin $\mathrm{C}$ type combined methylmalonic aciduria and homocystinuria. Zhongguo Dang Dai Er Ke Za Zhi 17: 769-774, 2015 (In Chinese).

25. Krueger JM, Piantino J, Smith CM, Angle B, Venkatesan C and Wainwright MS: A treatable metabolic cause of encephalopathy: Cobalamin $\mathrm{C}$ deficiency in an 8-year-old male. Pediatrics 135: e202-e206, 2015.

26. Chen Z, Yuan L, Zhang G and Shen Y: Renal damage caused by renal damage in 2 cases of children. Chin J Prac Pediatr 25: 73-74, 2010 (In Chinese). 